Revue européenne des sciences sociales

European Journal of Social Sciences

XLII-129 | 2004

La sociologie durkheimienne : tradition et actualité

\title{
Les éternels retours. Notes sur les cycles de mode
}

\section{Alexandre Gofman}

\section{OpenEdition}

\section{Journals}

Édition électronique

URL : http://journals.openedition.org/ress/392

DOI : $10.4000 /$ ress.392

ISSN : 1663-4446

\section{Éditeur}

Librairie Droz

\section{Édition imprimée}

Date de publication : 1 mars 2004

Pagination : 135-144

ISBN : 2-600-00941-8

ISSN : 0048-8046

Référence électronique

Alexandre Gofman, «Les éternels retours. Notes sur les cycles de mode », Revue européenne des sciences sociales [En ligne], XLII-129 | 2004, mis en ligne le 05 novembre 2009, consulté le 19 avril 2019. URL : http://journals.openedition.org/ress/392 ; DOI : 10.4000/ress.392 


\section{Alexandre GOFMAN}

\section{LES ÉTERNELS RETOURS. NOTES SUR LES CYCLES DE MODE}

Dans les travaux de sociologie de la mode on souligne souvent que la mode et le vêtement sont choses différentes et que la mode n' «habite» pas uniquement dans le second. En même temps, on le sait mais on s'en souvient rarement, le «champ » du vêtement est régi par bien d'autres mécanismes socioculturels que la seule mode. Néanmoins, c'est sur l'habillement que les sociologues de la mode continuent le plus souvent de baser leurs recherches et d'en puiser des exemples. D'un côté, on peut trouver cela tout à fait naturel et bien fondé, car c'est justement dans ce domaine que la mode fonctionne par excellence. Mais d'autre côté, c'est précisément ici que le phénomène de la mode est le moins susceptible d'être identifié, isolé et séparé de ce qui n'est pas la mode proprement dite. Celle-ci s'y mêle en effet avec d'autres phénomènes à tel point qu'il est parfois impossible de la distinguer de ces derniers. C'est pourquoi, dans le cas de bien des recherches dont le titre comporte le terme de «mode», on croit étudier la mode mais on étudie en réalité quelque chose d'autre, tandis que la mode elle-même reste insaisissable.

Les travaux que Philippe Besnard et ses collaborateurs ont consacrés à la mode constituent une des rares et heureuses exceptions à cette règle ${ }^{1}$. Ces auteurs considèrent en effet les prénoms comme des biens de mode et le choix de cet objet est tout à fait novateur dans l'histoire des recherches sur la mode. Il faut souligner en outre qu'il s'agit bien de sociologie et non de l'anthroponymie traditionnelle ou, a fortiori, de la mystique des prénoms, approches que l'on rencontre plus ou moins fréquemment. Les auteurs voient (selon moi à juste titre) un des avantages de ce choix dans le fait que, à la différence des autres biens de mode, les prénoms sont donnés gratuitement, et la mode se trouve donc ici en un état plus «pur» que dans d'autres domaines où l'aspect financier, le coût du bien, empiètent sur la mode proprement dite ${ }^{2}$. En effet, s'agissant d'autres objets de consommation tels que le vêtement ou, plus encore, les produits de luxe, il est souvent très difficile de

\footnotetext{
Voir notamment: Besnard Ph., «Pour une étude empirique du phénomène de mode dans la consommation des biens symboliques: le cas des prénoms », Archives européennes de sociologie, 20 (2), 1979, 343-351; Besnard Ph. et Desplanques G., Un prénom pour toujours. La cote des prénoms hier, aujourd' hui et demain, Paris, Balland, 1986; La cote des prénoms en 1994, ibid., 1993; La cote des prénoms en 1997, ibid., 1996; La cote des prénoms en 1999, ibid., 1998; «Les catégories socioprofessionnelles à l'épreuve de la stratification temporelle des goûts », Revue française de sociologie, 40 (1), 1999, 97-109; Besnard Ph. et Grange C., «La fin de la diffusion verticale des goûts? (Prénoms de l'élite et du vulgum)», L'Année sociologique, 43, 1993, 269294.

2 Voir Besnard Ph. et Desplanques G., La cote des prénoms en 1994, p. 10; La cote des prénoms en 1997, p. 14.
} 
distinguer entre les valeurs de mode et les valeurs économiques qui leurs sont extérieures mais qui se confondent avec elles, ce qui complique le problème déjà mentionné de la spécification de la mode comme objet de recherche ${ }^{3}$.

Évidemment, en analysant leur objet, Philippe Besnard et ses collaborateurs ne peuvent pas faire l'économie d'une définition de la mode. On en trouve dans leurs textes deux versions très proches l'une de l'autre. Dans un cas, la mode est traitée comme «la transformation, à tendance cyclique, du goût collectif » ${ }^{4}$; dans l'autre, comme «une diffusion sociale verticale dans le mouvement de transformation des goûts à tendance cyclique $»^{5}$.

En ce qui concerne la diffusion verticale comme trait distinctif de la mode, l'attitude des auteurs est double et quelque peu ambiguë. Tout d'abord, ils affirment que «le trajet social des prénoms ne contredit pas les explications classiques de la diffusion verticale de la mode $»^{6}$. Il s'agit là d'un modèle qui trouve son origine dans les travaux de Herbert Spencer, Gabriel Tarde et Georg Simmel, et qui, dans la sociologie anglophone, a reçu le nom de «trickle down effect»... Mais, selon Besnard et ses coauteurs eux-mêmes, ce modèle convient de moins en moins à la société française actuelle; le titre de l'un de leurs articles - «La fin de la diffusion verticale des goûts?» - est à cet égard très significatif. À côté du mouvement vertical des biens de mode comme facteur de leurs transformations, ils en distinguent un autre, à savoir «le degré de sociabilité» («la fréquence des rapports interpersonnels ou des relations sociales $»^{7}$ ) et, par là même, le flux horizontal où «un prénom - ou n'importe quel bien de mode - se propage aussi comme une maladie contagieuse $»^{8}$. Ils soulignent la moindre emprise du modèle de diffusion verticale, ce qui contredit évidemment l'utilisation de ce dernier caractère comme trait distinctif de la mode.

Il faudrait ajouter à cela que la diffusion verticale à l'époque moderne et «post-moderne» suppose un mouvement des biens de mode non seulement «de haut en bas » de l'échelle sociale mais aussi un mouvement de sens inverse, et que la «contagion» se produit non seulement de façon horizontale mais aussi de façon verticale «ascensionnelle». En général, les résultats des recherches de Besnard et ses collègues confirment de manière convaincante que le modèle de «trickle down

Pourtant, l'affirmation des auteurs selon laquelle «l'utilité objective du produit» empêche le fonctionnement de la mode me semble douteuse et ce doute est renforcé par l'exemple choisi pour soutenir cette affirmation: «... L'aspirine ne se démode pas malgré son énorme diffusion...» (Ibid.). Tout d'abord, si, aujourd'hui, l'aspirine ne s'est pas encore démodée (il semble que ce soit bien le cas), on ne peut pas être sûr que cela n'arrivera pas dans un futur plus ou moins proche, malgré «l'utilité objective» de ce produit. On connaît beaucoup de biens de mode « objectivement utiles » qui se sont malheureusement démodés. En général, «l'utilité objective», ainsi que «l'inutilité objective» (ou «subjective»), peut elle-même être à la mode et donc, par cela même, se démoder.

4 Besnard Ph. et Desplanques G., La cote des prénoms en 1994, p. 8; La cote des prénoms en 1997, p. 12.

5 Besnard Ph. et Desplanques G., «Les catégories socioprofessionnelles à l'épreuve de la stratification temporelle des goûts », art. cit., p. 97.

6 Besnard Ph. et Desplanques G., La cote des prénoms en 1994, p. 273; La cote des prénoms en 1997, p. 295.

Ibid., p. 274 ; ibid., p. 296.

Ibid. 
effect» n'est plus du tout suffisant aujourd'hui pour décrire et expliquer la mode en tant que telle. Ce que ce modèle décrit et explique bien c'est, à mon avis, le phénomène du snobisme mais celui-ci et la mode, bien qu'ils se recoupent parfois, ne sont pas synonymes.

Le caractère cyclique, d'après nos auteurs, est un autre trait distinctif de la mode. À la différence du premier trait, à savoir la diffusion verticale, ils le considèrent comme un phénomène indiscutable et universel et l'analysent de manière tout à fait détaillée. Ils distinguent les différentes périodes des cycles de mode des prénoms, en les spécifiant et en les présentant sous forme de courbes de popularité... Les stades de ces cycles sont notamment qualifiées de «précurseur», «pionnier», «dans le vent», «hyperconformiste et conformiste», «à la traîne», «démodé», «désuet», «rare», «dans le flux», «plutôt conformiste», «en reflux », «classique » ${ }^{9}$. Les résultats de ces recherches concernant les cycles de mode dans le domaine des prénoms sont fort intéressants car ils permettent d'affiner nos représentations dans ce domaine. C'est cette question des cycles de mode que je voudrais discuter de façon un peu plus ample, afin de préciser quelques points importants de la problématique.

L'aspect cyclique est un des deux aspects - ou des deux dimensions - du changement de mode. L'autre dimension est l'innovation de mode qui consiste, c'est évident, en ce qu'à la place de certains biens de mode («démodés») en apparaissent d'autres («à la mode»). Cet aspect est étroitement lié à l'aspect cyclique et se transforme de manière imperceptible en celui-ci. En effet, chaque «mode» vieillit avec le temps, l'innovation cesse d'être innovation et l'on découvre que son existence a achevé un cycle qui cède alors la place à un autre cycle. En général, les innovations et les cycles constituent deux aspects inséparables du changement de mode. Dans une certaine mesure, on a affaire au même processus de changement de mode considéré sous différents angles. On peut dire que les innovations de mode peuvent être considérées comme des changements des cycles de mode, et que les cycles de mode constituent une série d'innovations achevées.

Rappelons que le mot «cycle» provient du mot grec kuklos (cercle) et signifie une circulation plus ou moins régulière et achevée, effectuée par un phénomène pendant une certaine période. Il y a déjà longtemps que l'on a constaté le caractère cyclique du mouvement des biens de mode (que l'on appelle «les modes» au pluriel dans le langage courant $)^{10}$. Aujourd'hui, le concept de «cycle de mode», comme celui de «cycle de vie d'un produit», est largement utilisé par les spécia-

Ibid., p. 64-67; ibid., p. 68-71.

${ }^{10}$ Voir notamment Kroeber A., «Order in changes of fashion» (1919), in Kroeber A., The nature of culture, Chicago, University of Chicago Press, 1952; Richardson J., Kroeber A., «Three centuries of women's dress fashions: a quantitative analysis » (1940) in Kroeber A., The nature of culture, op. cit.; Nystrom P. H., Economics of fashion, New York, Ronald Press, 1928, p. 18; Young A. B., Recurring cycles of fashion. 1760-1937 (1937), $2^{\text {nd }}$ ed. New York, Cooper Square Publ., 1966; Meyersohn R., Katz E., «Notes on a natural history of fads », American Journal of Sociology, 62 (6), 1957, 594-601; Wasson C. R., Dynamic competitive strategy and product life cycle, St. Charles (Ill.), Challenge Books, 1974; Robinson D. E., «Style changes: cyclical, inexorable and foreseeable», Harvard Business Review, 53 (6), 1975, 121-131; Robinson D. E., «Fashions in shaving and trimming of the beard: The Men of the Illustrated London News. 1842-1972», American Journal of Sociology, 81 (5), 1976, 1133-1141; Sproles G. B., « Analyzing fashion life cycles - principles and perspectives », Journal of marketing, 45 (4), 1981, 116-124. 
listes du marketing et du design industriels, ainsi que par les scientifiques. Ils analysent l'essence des cycles de mode, étudient les particularités et la succession de leurs phases, calculent la durée des cycles et de leurs phases, font des pronostics sur tels ou tels paramètres des «modes", etc. Dans ces études, les chercheurs ont obtenu des résultats substantiels. Néanmoins, il reste aujourd'hui encore beaucoup de flou dans ce domaine. Bien que tout le monde reconnaisse l'existence de cycles de mode, les thèses sur ce sujet continuent d'être assez confuses et contradictoires. Évidemment, cela s'explique en partie par la complexité même de ce sujet, par sa versatilité, par la mutabilité de ses limites spatiales et temporelles. Pourtant, il semble qu'il y ait aussi là-dessous un problème d'ordre théorique.

Le fait est qu'on se heurte à la polysémie non évidente de l'expression «cycle de mode». En fait, il y a deux espèces différentes de cycles dans la mode et les spécialistes ont affaire tantôt à l'une, tantôt à l'autre, tantôt aux deux, sans prendre garde à leur différence ${ }^{11}$.

La première espèce (nommons-là «cycles de type I») caractérise le fonctionnement, l'évolution et le changement des «modes «comme telles - indépendamment ou en faisant abstraction de l'échelle et des particularités de leur diffusion. Ces cycles concernent tous les passages et oscillations, plus ou moins réguliers, d'une «mode » à l'autre et le retour à la précédente dans des intervalles de temps déterminés. On considère, par exemple, comme autant de cycles de ce type les oscillations de certains paramètres du style d'un vêtement à partir les uns des autres: les passages de l'étroit au large, de l'ouvert au fermé, du long au court (et vice versa), etc. On y rapporte aussi certains rythmes de l'alternance des couleurs, des tissus et autres aspects du vêtement. On trouve aussi de tels va-et-vient ou de tels rythmes dans d'autres domaines de la culture. Dans les recherches sur le vêtement, on considère que tel ou tel trait stylistique se transforme à partir d'un point donné et jusqu'à un certain terme (par exemple, du plus court jusqu'au plus long), après quoi le mouvement s'inverse. Ces oscillations entre certaines limites définies et au cours d'une période déterminée, forment le cycle de type I. On observe souvent ces oscillations non pas à propos d'un seul trait mais dans un ensemble ou une combinaison de traits stylistiques considérés comme les plus importants. Sont considérés comme cycles des changements de «modes» de différentes «longueur» et durée; par exemple, il peut s'agir d'un cycle allant du plus court au plus long ou un cycle «de retour» qui correspond à un mouvement du plus long au plus court - et de nouveau au plus long, etc.

La plupart des recherches des cycles de type I sont basées sur l'analyse des revues et magazines de modes. On suppose de façon implicite que ces publications présentent de manière assez fidèle les biens de mode ( «les modes ») qui agissent dans la vie «réelle»; le degré de diffusion de ces «modes» est considéré comme une grandeur assez constante et considérable.

La deuxième espèce de cycles de mode (nommons-les «cycles de type II» concerne plutôt non pas les changements des «modes» comme telles mais leur diffusion, leur acceptation par les participants à la mode. En ce cas, le caractère

Voir, à ce sujet, mes propres conclusions: Gofman A. B., Moda i liudi. Novaia teoria mody $i$ modnogo poviedienia (La Mode et les gens. Une nouvelle théorie de la mode et du comportement de mode), Moscou, Naouka, 1994 (2éd., Moscou, Gnom i D, 2000, chap. 3). 
cyclique consiste en ce qu'après la promotion et (ou) l'acceptation d'une certaine mode par une faible minorité d'individus s'ensuit sa reconnaissance par une majorité; ensuite, le nombre d'adhérents à cette «mode» diminue de nouveau jusqu'à son remplacement par une autre «mode» qui devient peu à peu populaire, etc. Si, dans les recherches des cycles de type I, on prend comme grandeur plus ou moins constante et connue (considérable) le degré et le caractère de la diffusion des «modes» en se concentrant sur l'étude des changements de chacune d'elles, dans l'analyse des cycles de type II, au contraire, chaque «mode» devient une grandeur plus ou moins constante et la variabilité est principalement observée dans le nombre et les particularités de ses adhérents, c'est-à-dire dans le degré de popularité de telle ou telle «mode». On peut dire que ces cycles consistent dans les changements de modes inchangées ayant une popularité changeante. Dans les biens de consommation courante, le cycle de type II est une variété du «cycle de vie du produit» (notion largement utilisée dans le marketing), à savoir du produit auquel sont attribuées des significations de mode ou, tout simplement, qui est «à la mode».

Il existe différents points de vue sur le caractère des cycles de mode, leurs régularités et la possibilité de les prévoir. Les différences concernent l'une et l'autre des deux espèces de cycles de mode que nous avons distinguées. Certains analystes soulignent le caractère conventionnel du concept du cycle appliqué à la mode, et la différence fondamentale entre les cycles de mode et de ceux de la nature. Ils nient l'existence de rythmes nets dans le mouvement des «modes» et, par conséquent, la possibilité d'en effectuer des mesures et des prévisions précises et adéquates. D'autres insistent sur la stricte régularité des changements et de l'alternance des «modes » et, par là même, sur la nécessité et sur la possibilité de leur analyse quantitative et de pronostics précis. On a fait notamment beaucoup de tentatives pour déterminer exactement la durée, la vitesse et les périodes des «retours» de certains traits stylistiques du vêtement considérés comme les plus importants.

A. Kroeber et J. Richardson, dans leur ouvrage classique, ont analysé les changements périodiques dans le vêtement féminin européen du XVII ${ }^{\mathrm{e}}$ siècle aux années $30 \mathrm{du} \mathrm{XX}^{\mathrm{e}}$ siècle selon six paramètres stylistiques. Comme source d'analyse quantitative, ils ont utilisé les journaux de mode. Ils mettent en relief un cycle principal de cent ans au cours duquel se manifestent des oscillations des traits stylistiques qu'ils ont choisi, d'un extrême à l'autre. Le caractère cyclique le plus important est d'ailleurs, d'après ces auteurs, celui de l'intensité des changements stylistiques. Kroeber et Richardson sont très prudents dans leurs conclusions générales à propos du caractère cycliques des changements de mode, et ils ne souhaitent pas donner à leurs résultats une importance universelle $\mathrm{e}^{12}$.

Un autre chercheur américain, D. Robinson, est beaucoup plus catégorique dans ses affirmations sur les cycles de mode; le titre de son article - «Changements de style: cycliques, inexorables et prévisibles » - est à cet égard très caractéristique ${ }^{13}$. D'après lui, les cycles de mode dans différents domaines, à savoir le

12 Voir Richardson J., Kroeber A., «Three centuries of women's dress fashions: a quantitative analysis », art. cit., p. 370-371.

${ }_{13}$ Robinson D. E., «Style changes: cyclical, inexorable and foreseeable», art. cit. 
vêtement, la barbe et la moustache (ou leur absence), le design des voitures et le style de vie en général durent à peu près 100 ans. En se basant sur l'étude des photos d'hommes publiées dans le magazine d'élite britannique Illustrated London News entre 1842 et 1972, il a effectué un calcul annuel comparatif de cinq caractéristiques morphologiques de la barbe et de la moustache - à savoir les favoris, les favoris avec moustache, la moustache seulement, la barbe et le visage entièrement rasé. Ses données sur l'alternance de ces caractéristiques correspondent, à son avis, aux mêmes régularités stylistiques que celles établies par Kroeber et Richardson ${ }^{14}$.

D'après Robinson, la régularité de ces cycles est si nette qu'ils sont insensibles aux changements technologiques, économiques, politiques, etc., pour autant que la tendance de la mode en question soit orientée dans un sens déterminé. De là s'ensuivent des conclusions d'ordre pratique pour le design et le marketing. Même pour les pronostics à court terme, le changement annuel peut et doit équivaloir à peu près à $2 \%$ en moyenne, en tenant compte de la durée séculaire d'un cycle complet et du changement maximum d'une tendance de style 50 ans après sa naissance. Selon cet auteur, il faut concrétiser et détailler ces affirmations générales sur les cycles de mode ${ }^{15}$ et c'est ici que réside, à mon avis, le problème le plus important et le plus difficile à résoudre.

Un exemple typique (et assez impressionnant) d'une analyse statistique scrupuleuse des cycles de type I est fourni par la recherche classique d'Agnès Brooks Young: Les cycles récurrents de la mode.1760-1937 (1937; 2ème éd. 1966). Young a analysé les évolutions du vêtement féminin durant la période 1760-1937 en Europe et en Amérique, en déterminant la mode annuelle dominante. Elle attache une importance particulière aux changements stylistiques de la forme de la jupe et en distingue trois types fondamentaux: le type «derrière rebondi» («back-fullness type»; l'auteur préfère cette expression à celle de «tournure» car cette dernière a, à son avis, une signification plus étroite dans l'histoire de l'habillement), le type «tubulaire»(«tubular type»), le type «en cloche» («bellshaped type»; ici encore elle préfère ce terme à ceux de paniers ou de crinoline pour la même raison que dans le premier cas). Ces trois types prédominent successivement pendant des périodes déterminées. Chacun d'eux prédomine, après avoir supplanté les deux autres, durant à peu près un tiers de siècle. Un cycle entier de changement de ces trois types dure 100 ans. Dans la période envisagée, l'auteur distingue les cycles suivants: 1760-1795 (36 ans de type «derrière rebondi »); 1796-1829 (34 ans de type «tubulaire»); 1830-1867 (38 ans de type «en cloche »); 1868-1899 (32 ans de type «derrière rebondi »); 1900-1937 (38 ans de type «tubulaire»). ${ }^{16}$

Young remarque que le manque de sources ne lui permet pas de faire une analyse annuelle de l'époque antérieure, mais elle fait l'hypothèse que ces cycles ont dû être identiques. On peut supposer que dans les années 1720-1760 eut lieu un cycle de jupe «en cloche», comme on peut le voir dans la peinture de Watteau, de Boucher, de Leclerc ou de Fragonard. De telles régularités permettraient de

\footnotetext{
${ }^{14}$ Robinson D. E., «Fashions in shaving and trimming of the beard», art. cit.

15 Robinson D. E., «Style changes...», p. 131.

16 Voir Young A. B., Recurring cycles of fashion., op. cit., p. 45-133.
} 
prédire exactement les modes vestimentaires : le nouveau cycle sera toujours celui qui précédait les deux autres, ou encore celui qui dominait un siècle auparavant. Et comme les changements annuels sont toujours graduels, le designer pourrait être sûr qu'il ne lui faut pas compter sur un changement brusque de la mode. La connaissance des régularités présentées par l'évolution des modes ne lui suggèrera pas toujours les innovations qu'il convient d'introduire mais elle pourra au moins le prévenir contre de fausses attentes.

Mais pourquoi le vêtement féminin change-t-il? L'auteur divise la question en deux parties: 1) Pourquoi des changements permanents et à l'évidence inutiles font-ils, annuellement, passer de mode les «modes» de l'année précédente? 2) Pourquoi ces changements se produisent-ils selon les cycles réguliers qui viennent d'être décrits?

À la première question, Young donne une réponse purement psychologique en imputant les changements de mode annuels à des besoins psychiques profonds de nouvelles expériences et de reconnaissance des autres ${ }^{17}$. Elle explique l'existence et la durée de chaque cycle (un tiers de siècle) par le fait que c'est probablement le temps nécessaire à la consolidation, à l'évolution et au déclin de chacun des types fondamentaux mis en évidence. En ce qui concerne l'ordre des « retours » de ces styles, elle l'explique par la différence d'attitude des gens envers le passé selon qu'il est récent ou lointain. Elle en tire une distinction entre quatre périodes dans nos attitudes envers n'importe quel style, successivement considéré comme «dérisoire », «toléré», «désirable» et, enfin, «hautement valorisé ». La durée de ces périodes exercerait une influence sur l'ordre du retour des cycles. L'auteur affirme enfin que ces quatre périodes n'existent pas seulement dans le domaine du vêtement féminin: «Probablement, toutes les formes de l'expression artistique, la peinture, la décoration intérieure, l'architecture et l'artisanat passent par ces quatre phases... Il est probable que la pensée et les attitudes intellectuelles du passé sont également évaluées selon ce même processus ${ }^{18}$.

Young reconnaît l'insuffisance de ces explications mais elle remarque en même temps que la connaissance des causes n'est pas toujours nécessaire aux prédictions pratiques; de plus, note-t-elle, il existe beaucoup de cycles, économiques ou naturels, dont l'existence et les caractéristiques sont établies mais qui n'ont pas, à ce jour, reçu d'explication satisfaisante.

On pourrait citer d'autres tentatives, toutes fort différentes, de détermination et de prédictions des cycles de la mode vestimentaire. L'évaluation des tentatives de mise en évidence des caractéristiques et la durée des cycles de type I conduit à souligner qu'elles s'inscrivent généralement dans les cadres généraux de multiples recherches relatives aux diverses manifestations universelles des rythmes dans la nature, dans la société et dans la culture. Parmi les recherches les plus notables, on peut citer celles d'Alexandre Tchijevsky sur le caractère rythmique des épidémies et d'autres phénomènes dans leur rapport à l'activité solaire, les travaux de Nikolaï Perna sur la théorie du rythme et l'influence des rythmes biologiques sur l'activité créatrice de l'homme. On étudiait les rythmes dans les processus du travail et dans les arts temporels (poésie, musique, danse). Les

17 Ibid., p. 166-171.

18 Ibid., p. 176. 
recherches des cycles dans ces différents domaines sont étroitement liées à celles des rythmes, elles s'entrecroisent en partie avec celles-ci ou les pénètrent. On étudiait notamment les cycles en économie (les travaux de Nikolaï Kondratiev, etc.), en histoire de la culture (les travaux de Pitirim Sorokin, etc.), en mythologie (Mircea Eliade), en politique (les études du comportement électoral, de l'alternance des partis politiques au pouvoir), etc.

Dans ces recherches, toutes fort intéressantes et parfois remarquables, les tentatives de définition des cycles de mode paraissent fructueuses; beaucoup de leurs résultats s'avèrent importants tant du point de vue scientifique que du point de vue pratique. Toutefois, les acquis relatifs à l'étude des cycles de type I demeurent assez modestes, et beaucoup de points y restent vagues. On observe des divergences considérables dans les critères stylistiques choisis pour distinguer ces cycles, dans l'appréciation de la durée de ces derniers et de la nature de leur alternance. On peut s'interroger sur le fondement du choix de tel ou tel critère pour caractériser un cycle.

L'affirmation selon laquelle l'évolution et l'alternance des «modes» serait analogue à celles des saisons ou des autres flux et reflux naturels peut être interprétée au moins comme une exagération polémique. On sait bien que les temps de la nature et de la culture ne coïncident pas, que la vitesse et les rythmes des processus sociaux varient selon les époques historiques et les milieux socio-culturels. Il est donc important de prendre en considération que les magazines de mode, sur lesquels sont souvent basées les recherches des cycles de mode, sont loin de nous fournir une représentation adéquate de la «vie» réelle des «modes» et, partant, de leurs cycles de vie. De là, la difficulté de déterminer les limites exactes, spatiales et temporelles, des cycles de type I.

Non seulement, les interprétations mécanistes de ces cycles peuvent provoquer et provoquent de graves erreurs de pronostic (par exemple, la prévision faite par Agnès Brooks Young sur la période postérieure à 1937); elles peuvent aussi exercer une influence négative sur l'activité des «créateurs » de mode. Le rôle du designer est en effet, dans ce cas, aussi simple que passif; au lieu de faire la mode, il peut se contenter de savoir quand et à quel moment il lui est nécessaire de réactiver quelle «mode» particulière, en la «modernisant» un peu.

Les «retours» stylistiques, de même que les autres «retours» (cycles de type I), existent réellement; il faut seulement prendre garde de ne pas leur attribuer, sous prétexte de «régularités nécessaires», des caractéristiques universelles applicables n'importe où et n'importe quand. C'est là une situation où la statistique conduit facilement à la mystique. La représentation du changement des «modes » comme un simple ensemble de répétitions, comme une suite de cycles où il n'y a rien de nouveau sous le soleil, est, à mon avis, aussi fausse que la représentation opposée de la mode comme couloir ascendant de nouveauté où le «nouveau » est constamment remplacé par «plus nouveau » encore.

Lorsque les «modes» sont assez éloignées de nous dans le temps pour avoir été oubliées, c'est-à-dire «nouvelles de nouveau», alors leur retour est possible, sans être toutefois obligatoire ${ }^{19}$. Nous pouvons constater plus ou moins exacte-

Cet «oubli social» dans la mode est renforcé par de nombreux facteurs, à savoir par les flux des innovations, la disparition des traditions, etc. A ce propos, il faut aussi noter l'importance du changement des générations qui suffit pour que le retour aux modèles culturels du passé pour une 
ment quand cet «oubli social» se produira, mais nous pouvons affirmer avec beaucoup moins d'exactitude ce qui, précisément, «reviendra» car la diversité et la richesse des formes culturelles «oubliées» (probablement pas pour toujours) sont vraiment infinies. Aussi ce «retour» peut-il n'avoir pas lieu du tout à cause d'innovations proprement dites, c'est-à-dire sans précédents historiques.

Certes, il est important d'essayer d'analyser et de prévoir la venue de ces cycles de type I. Pourtant, dans des domaines professionnels, il ne semble pas que l'activité pronostique puisse consister à calculer ce qui «sera à la mode» telle ou telle année - comme si les modes n'étaient pas faites par les hommes mais tombaient du ciel. Il est donc important de prédire non pas les «modes» comme telles mais les réalités qui se trouvent «derrière » ou «sous » elles, c'est-à-dire les tendances, les stratégies, les valeurs, les technologies, etc., ainsi que les problèmes, besoins et perspectives qu'elles induisent. Quant aux «modes » ellesmêmes, les vrais créateurs ne les prédisent pas en calculant quand on pourrait revenir à des formes culturelles préexistantes, mais ils les font, en tenant compte du présent et du futur et en s'inspirant plus ou moins consciemment des traditions et des tendances culturelles.

Pour ce qui est des cycles de type II, qui concernent la dynamique de la diffusion des certains biens de mode, les spécialistes des différents domaines du marketing aspirent souvent à déterminer les caractéristiques de ces cycles pour planifier et promouvoir certains produits. Malgré une grande diversité dans la périodisation de ces cycles, il s'agit, d'une manière ou d'une autre, des stades de la naissance et de l'enfance d'une «mode» (un minimum d'adhérents), de la maturité et du sommet (un maximum d'adhérents), du déclin (de nouveau un minimum d'adhérents), puis de la mort avec son remplacement par une autre «mode» (rappelons, qu'il peut s'agir de n'importe quel bien, produit, modèle culturel, trait stylistique, etc.). Pendant le stade de sa diffusion maximum, au sommet de sa popularité, un bien «à la mode» est pour ses adhérents non seulement le plus beau mais aussi le plus «naturel», le plus «normal», le plus «confortable», le plus « utile», etc. ${ }^{20}$ Il semble alors qu'il en a toujours été ainsi, qu'il est impossible qu'il en soit autrement et que, probablement, cela sera toujours ainsi. Notons à ce propos que le terme «classique», couramment employé dans le langage des designers et consommateurs pour qualifier quelque chose de plus ou moins évident, clair et stable (par exemple dans la phrase définitive: «Je suis partisan du style classique !»), correspond en fait à un phénomène essentiellement mouvant et variable. Son sens varie selon les moments de l'histoire culturelle et de la mode. En général, on peut observer que le style «classique» est la version la moins outrée, la moins exagérée, la moins «récente» (ou perçue et interprétée comme telle) de tel ou tel style à la mode, et c'est pourquoi son contenu diffère

génération donnée puisse apparaître comme une innovation et une «nouvelle» mode. Sous ce rapport, la coïncidence qu'on trouve dans plusieurs recherches sur l'alternance de trois cycles au cours d'un siècle, d'une part, et l'idée socio-démographique des générations généalogiques (grands-pères, pères, petits-fils), trois par siècle également, d'autre part, ne paraît pas fortuite. Pourtant, le facteur générationnel dans les cycles de type I ne peut pas être considéré comme unique ou décisif, car ceux-ci sont déterminés par tout un ensemble de causes d'ordre social et culturel.

20 À ce moment précis, «l'utilité objective» de l'aspirine peut paraître la plus grande. 
d'une période à l'autre. Par exemple, lorsque l'étroit est à la mode, le «classique» sera ce qui n'est pas trop étroit; lorsque c'est le large, ce qui n'est pas trop large, etc.

La tendance générale constatée dans les sociétés modernes et «postmodernes » est à la réduction de la durée des cycles de type II de beaucoup de biens de consommation, à l'accélération de leur usure morale (ainsi que physique). C'est cette tendance même que démontrent les recherches de Philippe Besnard et ses collaborateurs dans le domaine des prénoms.

Ce phénomène de la réduction de la durée des cycles de type II suscite parmi les créateurs tantôt de l'enthousiasme tantôt de l'inquiétude et la déception. On peut pourtant, aujourd'hui, constater que les prévisions d'Alvin Toffler dans son fameux ouvrage de 1970 (selon lequel nous entrerions dans l'ère du provisoire fabriqué par des méthodes provisoires afin de satisfaire des besoins provisoires ${ }^{21}$ ) ne se sont que partiellement réalisées. Il n'y a certes rien d'éternel sur cette terre mais ces déclarations sur le triomphe total des principes de l'éphémère et de la nouveauté dans les rapports entre l'homme et les choses étaient évidemment exagérées, ainsi qu'on peut le constater aujourd'hui, plus de trente ans après.

Il est tout à fait évident que les modes changent, mais la Mode comme telle est un phénomène constant. À mon avis, le caractère cyclique, tout comme le caractère innovateur, ne peut être généralement considéré comme le seul trait spécifique de la mode. D'un côté, on le sait, il y a beaucoup de phénomènes cycliques qui ne sont pas des modes. D'un autre côté, ce caractère cyclique n'est pas un indice distinctif suffisant de la mode. Il doit y avoir quelque chose de plus pour qu'on puisse identifier ce phénomène de la mode. Quel est ce «quelque chose»? C'est au moins un sujet pour d'autres notes ou articles...

Département de sociologie générale Haut Collège d'Économie, Moscou agofman@iname.com

Toffler A., Future shock, New York, Random House, 1970. Sur ce même sujet de «l'éphémérisation» de la consommation, voir: Lipovetsky G., L'Empire de l'éphémère. La mode et son destin dans les sociétés modernes, Paris, Gallimard, 1987. 